\title{
ON MONOTONICITY AND SUPERADDITIVITY PROPERTIES OF THE ENTROPY FUNCTION
}

\author{
S. S. DRAGOMIR ${ }^{1}$ and C. J. GOH ${ }^{2}$ \\ (Received 28 November 1998; revised 22 November 1999)

\begin{abstract}
We apply superadditivity and monotonicity properties associated with the Jensen discrete inequality to derive relationships between the entropy function of a probability vector and a renormalized arbitrary sub-vector. The results are extended to cover other entropy measures such as joint entropy, conditional entropy and mutual information.
\end{abstract}

\section{Introduction}

Let $\mathbf{X}$ be a real linear space, $C$ a convex subset and $f: C \rightarrow \mathbb{R}$ a real convex mapping. Suppose $\mathscr{I}$ is a finite nonempty set of natural numbers with $p_{i}>0$ and $\xi_{i} \in C$ for all $i \in \mathscr{I}$. Here and subsequently we write $P_{I}:=\sum_{i \in I} p_{i}$ for $I$ a nonempty subset of $\mathscr{I}$ (denoted by a single upper-case letter). Jensen's discrete inequality states that

$$
f\left(\frac{1}{P_{I}} \sum_{i \in I} p_{i} \xi_{i}\right) \leq \frac{1}{P_{I}} \sum_{i \in I} p_{i} f\left(\xi_{i}\right) .
$$

For some related results, see [2]. We write

$$
\begin{aligned}
S(C, \mathbb{R}) & :=\text { the linear space of all real functions on } C ; \\
2^{\mathscr{I}} & :=\text { the power set of } \mathscr{I}, \text { that is, the set of all subsets of } \mathscr{I} ; \\
\mathbf{p} & :=\left\{p_{i}\right\}_{i \in \mathscr{I}}, p_{i}>0 \quad \forall i \in \mathscr{I} ; \\
\mathscr{P}_{\mathscr{I}} & :=\left\{\mathbf{p}_{J}=\left\{p_{i}\right\}_{i \in J}, J \in 2^{\mathscr{J}}\right\}, \text { the set of all subvectors of } \mathbf{p} ; \\
\mathscr{X} & :=\left\{\left\{\xi_{i}\right\}_{i \in \mathscr{I}}, \xi_{i} \in C, \forall i \in \mathscr{I}\right\} ; \\
\mathscr{X}_{\mathscr{I}} & :=\left\{\xi_{J}=\left\{\xi_{i}\right\}_{i \in J}, J \in 2^{\mathscr{I}}\right\}, \text { the set of all subsets of } \mathscr{X} .
\end{aligned}
$$

\footnotetext{
'School of Communications and Informatics, Victoria University of Technology, PO Box 14428, Melbourne City MC, VIC 8001, Australia.

${ }^{2}$ Department of Mathematics, University of Western Australia, Nedlands, WA 6907, Australia.

(C) Australian Mathematical Society 2001, Serial-fee code 0334-2700/01
} 
In [2], Dragomir et al. considered the functional

$$
F: S(C, \mathbb{R}) \times 2^{\mathscr{J}} \times \mathscr{P}_{\mathscr{I}} \times \mathscr{X}_{\mathscr{I}} \rightarrow \mathbb{R}
$$

given by

$$
F=F\left(f, J, \mathbf{p}_{J}, \boldsymbol{\xi}_{J}\right)=\sum_{i \in J} p_{i} f\left(\xi_{i}\right)-P_{J} f\left(\frac{1}{P_{J}} \sum_{i \in J} p_{i} \xi_{i}\right) .
$$

Henceforth we assume that $\mathbf{p}$ and $\boldsymbol{\xi}$ are fixed, so that $F$ is essentially dependent on only the first two arguments, that is, $F\left(f, J, \mathbf{p}_{J}, \xi_{J}\right)=F(f, J)$. The following results were obtained in [2].

THEOREM 1.1. (i) As an index function on $2^{\mathcal{J}}, F(f, \cdot)$ is superadditive, that is, if $J, K \in 2^{\sigma}$ with $J \cap K=\emptyset$, then

$$
F(f, J \cup K) \geq F(f, J)+F(f, K) \geq 0 .
$$

(ii) As an index function on $2^{\mathscr{f}}, F(f, \cdot)$ is monotone, that is, if $J, K \in 2^{\mathscr{f}}$ and $K \subseteq J$, then

$$
F(f, J) \geq F(f, K) \geq 0
$$

COROLLARY 1.2. We have

(i) $\max _{J \in 2} F(f, J)=F(f, \mathscr{I}) \geq 0$;

(ii) for every $J \in 2^{\mathcal{J}}$ with $|J| \geq 2$,

$$
F(f, J) \geq \max _{i, j \in \mathcal{I}}\left[p_{i} f\left(\xi_{i}\right)+p_{j} f\left(\xi_{j}\right)-\left(p_{i}+p_{j}\right) f\left(\frac{p_{i} \xi_{i}+p_{j} \xi_{j}}{p_{i}+p_{j}}\right)\right] \geq 0 .
$$

In the special case $J_{k}=\{1,2, \ldots, k\}$, we denote $F\left(f, J_{k}\right)$ by

$$
F_{k}(f):=\sum_{i=1}^{k} p_{i} f\left(\xi_{i}\right)-P_{k} f\left(\frac{1}{P_{k}} \sum_{i=1}^{k} p_{i} \xi_{i}\right) \geq 0 .
$$

Here, as subsequently, $P_{k}:=\sum_{i=1}^{k} p_{i}$ for $k$ (a lower-case letter) some positive integer. We then have the following further corollary.

COROLlaRY 1.3. We have

(i) $\max _{J_{\in} J_{k}, J \neq \emptyset} F(f, J)=F_{k}(f) \geq 0$;

(ii) $F_{k}(f) \geq \max _{1 \leq i<j \leq k}\left[p_{i} f\left(\xi_{i}\right)+p_{j} f\left(\xi_{j}\right)-\left(p_{i}+p_{j}\right) f\left(\frac{p_{i} \xi_{i}+p_{j} \xi_{j}}{p_{i}+p_{j}}\right)\right] \geq 0$;

(iii) $F_{k}(f) \geq F_{k-1}(f) \geq F_{k-2}(f) \geq \cdots \geq F_{2}(f) \geq 0$. 
These superadditivity and monotonicity properties of the functional $F$ have application to bounds for entropy functions in information theory in the case of the convex function $f(\cdot)=-\log _{b}(\cdot)(b>0)$. Suppose a random variable $X$ has a finite range. The entropy of $X$ is a measure of the uncertainty associated with it. If some prior information allows us to restrict the possible outcome of $X$ to some subset of the original range, without any change in the relative probability distribution, then it is of interest to relate, by means of appropriate bounds, the uncertainty of the original random variable to that of the restricted one. In this paper some such bounds are established.

\section{Bounds for the entropy mapping}

Suppose $X$ is a discrete random variable having range $R=\left\{x_{i}, i \in \mathscr{I}\right\}$ and that $\operatorname{Pr}\left(X=x_{i}\right)=p_{i}>0(i \in \mathscr{I})$. Let $\mathbf{p}$ be the corresponding probability vector. The $b$-entropy (or more briefly the entropy) of $X$ is defined by

$$
H_{b}(X)=H_{b}(\mathbf{p}):=\sum_{i \in \mathscr{I}} p_{i} \log _{b}\left(1 / p_{i}\right)
$$

(see [3]). The first inequality of the following bound on $H_{b}(X)$ is well-known in information theory (see, for example, [3]). The second was established as Theorem 4.3 of [1].

THEOREM 2.1. The entropy of $X$ satisfies

$$
0 \leq \log _{b}|\mathscr{I}|-H_{b}(X) \leq \frac{1}{\ln b}\left[|\mathscr{I}| \sum_{i \in \mathscr{I}} p_{i}^{2}-1\right] .
$$

Furthermore, $H_{b}(X)=0$ if and only if $p_{i}=1$ for some $i$; and $H_{b}(X)=\log _{b}|\mathscr{I}|$ if and only if $p_{i}=1 /|\mathscr{I}|$ for each $i \in \mathscr{I}$.

Suppose we wish to relate the entropy of some renormalized sub-probability vector of $\mathbf{p}$ to that of $\mathbf{p}$. For $J \subseteq \mathscr{I}$, define a new random variable $X_{J}$ having range $R_{J}:=\left\{x_{i}, i \in J\right\}$ and probability distribution $\left\{p_{i}^{J}:=p_{i} / P_{J}>0, i \in J\right\}$. Put $\mathbf{p}_{J}=$ $\left\{p_{i}^{J}, j \in J\right\}$. The entropy of $X_{J}$ is then given by $H_{b}\left(X_{J}\right):=\sum_{j \in J} p_{j}^{J} \log _{b}\left(1 / p_{j}^{J}\right)$.

THEOREM 2.2. We have

$$
\begin{aligned}
\log _{b}|\mathscr{I}|-H_{b}(X) & =\max _{J \in 2^{\mathcal{S}}, J \neq \emptyset}\left\{P_{J}\left[\log _{b}|J|-H_{b}\left(X_{J}\right)\right]\right\} \\
& \geq \max _{1 \leq i<j \leq|\mathscr{\mathscr { S }}|}\left\{\left(p_{i}+p_{j}\right)\left[\log _{b} 2-H_{b}\left(X_{\{i, j]}\right)\right]\right\} \geq 0
\end{aligned}
$$


ProOF. We may express $H_{b}\left(X_{J}\right)$ as

$$
H_{b}\left(X_{J}\right)=\sum_{j \in J} \frac{p_{j}}{P_{J}} \log _{b}\left(P_{J} / p_{j}\right)=\log _{b} P_{J}+\frac{1}{P_{J}} \sum_{j \in J} p_{j} \log _{b}\left(1 / p_{j}\right)
$$

Also

$$
H_{b}\left(X_{\{i, j\}}\right)=\log _{b}\left(p_{i}+p_{j}\right)+\frac{1}{p_{i}+p_{j}} \sum_{\ell=i, j} p_{\ell} \log _{b}\left(1 / p_{\ell}\right)
$$

for all $i, j \in \mathscr{I}$.

Setting $f(\cdot)=-\log _{b}(\cdot)$ and $\xi_{i}=1 / p_{i}$ in Corollary 1.2 yields

$$
\begin{aligned}
\log _{b}|\mathscr{I}|-H_{b}(X) & \\
= & \max _{J \in 2^{\Im}, J \neq \emptyset}\left[P_{J}\left[\log _{b}\left(\frac{|J|}{P_{J}}\right)-\frac{1}{P_{J}} \sum_{j \in J} p_{j} \log _{b} \frac{1}{p_{j}}\right]\right] \\
& \geq \max _{1 \leq i<j \leq|\mathscr{I}|}\left[\left(p_{i}+p_{j}\right)\left[\log _{b}\left(\frac{2}{p_{i}+p_{j}}\right)-\frac{1}{p_{i}+p_{j}} \sum_{\ell=i, j} p_{\ell} \log _{b}\left(1 / p_{\ell}\right)\right]\right] \\
& \geq 0 .
\end{aligned}
$$

Inequality (2.1) follows from

$$
\log _{b}\left(\frac{|J|}{P_{J}}\right)-\frac{1}{P_{J}} \sum_{j \in J} p_{j} \log _{b}\left(1 / p_{j}\right)=\log _{b}|J|-H_{b}\left(X_{J}\right)
$$

and

$$
\log _{b}\left(\frac{2}{p_{i}+p_{j}}\right)-\frac{1}{p_{i}+p_{j}} \sum_{\ell=i, j} p_{\ell} \log _{b}\left(1 / p_{\ell}\right)=\log _{b} 2-H_{b}\left(\dot{X}_{\{i, j\}}\right)
$$

. We may define the random variables $X_{k}, k=2,3, \ldots,|\mathscr{I}|$, where $X_{|\mathscr{I}|}=X$ and $X_{k}$ has range $\left\{x_{1}, x_{2}, \ldots, x_{k}\right\}$ and corresponding probability vector $\left\{p_{1} / P_{k}, p_{2} / P_{k}, \ldots\right.$, $\left.p_{k} / P_{k}\right\}$. Corollary 1.3 yields the following.

COROLLARY 2.3. With the above assumptions,

$$
\begin{aligned}
\log _{b}|\mathscr{I}|-H_{b}(X) & \geq P_{|\mathscr{I}|-1}\left[\log _{b}(|\mathscr{I}|-1)-H_{b}\left(X_{|\mathscr{I}|-1}\right)\right] \\
& \geq \cdots \geq P_{2}\left[\log _{b} 2-H_{b}\left(X_{2}\right)\right] \geq 0 .
\end{aligned}
$$

Theorem 1.1 (i) can also be used to derive a relationship between the entropies of a probability vector and a pair of its mutually exclusive sub-probability vectors, after renormalization. We address the situation in which $\mathscr{I}$ decomposes properly into $J$ and $K$, that is, $\mathscr{I}=J \cup K$ with $J \cap K=\emptyset$ and $J, K \neq \emptyset$. 
THEOREM 2.4. Let the random variables $X_{J}$ and $X_{K}$ have ranges $R_{J}$ and $R_{K}$ respectively, with corresponding probability vectors

$$
\mathbf{p}^{J}=\left\{p_{i} / P_{J}, i \in J\right\} \quad \text { and } \quad \mathbf{p}^{K}=\left\{p_{i} / P_{K}, i \in K\right\}
$$

Then

$$
\log _{b}|\mathscr{I}|-H_{b}(X) \geq \sum_{A=J, K} P_{A}\left[\log _{b}|A|-H_{b}\left(X_{A}\right)\right] \geq 0
$$

Proof. Setting $f(\cdot)=-\log _{b}(\cdot)$ and $x_{i}=1 / p_{i}$ in Theorem 1.1 (i) provides

$$
\begin{aligned}
\log _{b}|\mathscr{I}|-H_{b}(X) & \geq \sum_{A=J, K}\left[\sum_{i \in A} p_{i} \log _{b} p_{i}+P_{A} \log _{b}\left(|J| / P_{J}\right)\right] \\
& =\sum_{A=J, K} P_{A}\left(\sum_{i \in A} \frac{p_{i}}{P_{A}} \log _{b} \frac{p_{i}}{P_{A}}+\log _{b}|A|\right)
\end{aligned}
$$

whence we have the desired result.

More interesting results can be deduced from Theorem 2.4. Define a new random variable $Z$ by $Z=1$ if $X \in R_{J}$ and $Z=0$ if $X \in R_{K}$. Then $\operatorname{Pr}(Z=1)=P_{J}$ and $\operatorname{Pr}(Z=0)=P_{K}$ with $P_{J}+P_{K}=1,|\mathscr{I}|=|J|+|K|$ and the entropy of $Z$ is given by

$$
H_{b}(Z)=\sum_{A=J, K} P_{A} \log _{b}\left(1 / P_{A}\right)
$$

We may rewrite (2.2) as

$$
\log _{b}\left(\frac{|\mathscr{I}|}{|J|^{P_{J}}|K|^{P_{K}}}\right) \geq H_{b}(X)-\sum_{A=J, K} P_{A} H_{b}\left(X_{A}\right) .
$$

The right-hand side may be expressed as

$$
\sum_{i \in \mathcal{I}} p_{i} \log _{b} \frac{1}{p_{i}}-\sum_{A=J, K} \sum_{i \in A} p_{i} \log _{b} \frac{\dot{P}_{A}}{p_{i}}=\sum_{A=J, K} P_{A} \log _{b} \frac{1}{P_{A}}=H(Z) .
$$

Since $0 \leq H(Z) \leq \log _{b} 2$, we have the following.

\section{COROLLARY 2.5 .}

$$
0 \leq H_{b}(X)-\sum_{A=J, K} P_{A} H_{b}\left(X_{A}\right)=H(Z) \leq \min \left\{\log _{b} 2, \log _{b}\left(\frac{|\mathscr{I}|}{|J|^{P_{1}}|K|^{P_{K}}}\right)\right\}
$$

If $|J|=|K|=|\mathscr{I}| / 2$ in (2.3), then $\log _{b}\left(|\mathscr{I}| /\left(|J|^{P_{J}}|K|^{P_{K}}\right)\right)=\log _{b} 2$ for all $P_{J}$ and $P_{K}$ such that $P_{J}+P_{K}=1$ and the last term in (2.3) is equal to $\log _{b} 2$ for all probability distributions. If $|J| \neq|K|$, the minimum is dependent on the relative 
magnitude of $|J|$ and $P_{J}$. In fact, a little analysis allows (2.3) to be expressed in a more explicit form. Suppose

$$
|J|<|\mathscr{I}| / 2<|K| .
$$

The difference between the two terms in braces in (2.3) is

$$
\log _{b}\left(\frac{|\mathscr{I}|}{|J|^{P_{J}}|K|^{P_{K}}}\right)-\log _{b} 2=P_{J} \log _{b} \frac{|K|}{|J|}+\log _{b} \frac{|\mathscr{I}|}{2|K|},
$$

which is linear in $P_{J}$, and by virtue of (2.4) we have

$$
\log _{b}(|K| /|J|)>0, \quad \log _{b}(|\mathscr{I}| /(2|K|))<0 .
$$

Thus the difference is negative when $P_{J}=0$ and positive when $P_{J}=1$. At the intermediate value of

$$
P_{J}=P_{J}^{*}=\frac{\log _{b}(2|K| /|\mathscr{I}|)}{\log _{b}(|K| /|J|)}
$$

the difference is zero. The case where $|J|>|\mathscr{I}| / 2>|K|$ is completely symmetrical. Thus the second inequality of Corollary 2.5 can be rewritten as

$$
H(Z) \leq \begin{cases}\log _{b}(|\mathscr{I}| /|K|)+P_{J} \log _{b}(|K| /|J|) & \text { if }\left(|J|<|K|, P_{J} \leq P_{J}^{*}\right) \\ & \text { or }\left(|J|>|K|, P_{J} \geq P_{J}^{*}\right), \\ \log _{b} 2 & \text { otherwise. }\end{cases}
$$

A further refinement to the lower bound of $H_{b}(Z)$ is possible. On applying the second inequality of Theorem 2.1 to the random variable $Z$, we obtain

$$
\dot{H}_{b}(Z) \geq \log _{b} 2-\frac{1}{\ln b}\left[2\left(P_{K}^{2}+P_{J}^{2}\right)-1\right]=\log _{b} 2-\frac{1}{\ln b}\left(P_{K}-P_{J}\right)^{2} .
$$

On combining (2.5) and (2.6), we obtain a tighter bound than that of Corollary 2.5.

COROLlary 2.6. We have

$$
\begin{aligned}
\max & \left\{0, \log _{b} 2-\frac{1}{\ln b}\left(P_{K}-P_{J}\right)^{2}\right\} \\
& \leq H_{b}(Z)=H_{b}(X)-\sum_{A=J, K} P_{A} H_{b}\left(X_{A}\right) \\
& \leq \begin{cases}\log _{b}(|\mathscr{I}| /|K|)+P_{J} \log _{b}(|K| /|J|) & \text { if }\left(|J|<|K|, P_{J} \leq P_{J}^{*}\right) \\
\log _{b} 2 & \text { or }\left(|J|>|K|, P_{J} \geq P_{J}^{*}\right), \\
& \text { otherwise. }\end{cases}
\end{aligned}
$$




\section{Extension to joint entropy}

Let $X, Y$ be random variables having respective ranges $R=\left\{x_{i}, i \in \mathscr{I}\right\}$ and $S=\left\{y_{j}, j \in \mathscr{L}\right\}$ and joint probability distribution $p(x, y)$. The joint entropy of $X$ and $Y$ is defined as

$$
H_{b}(X, Y):=\sum_{x \in R} \sum_{y \in S} p(x, y) \log _{b} \frac{1}{p(x, y)} .
$$

For the index subsets $J \in 2^{\mathscr{J}}$ and $K \in 2^{\mathscr{L}}$, we define a pair of new random variables $X_{J}$ and $Y_{K}$ having ranges $R_{J}:=\left\{x_{i}, i \in J\right\}$ and $S_{K}:=\left\{y_{i}, i \in K\right\}$ respectively. We introduce the notation

$$
P_{J K}:=\sum_{x \in R_{J}} \sum_{y \in S_{K}} p(x, y), \quad p_{J K}(x, y):=p(x, y) / P_{J K}
$$

and define the joint entropy of $X_{J}$ and $Y_{K}$ as

$$
H_{b}\left(X_{J}, Y_{K}\right):=\sum_{x \in R} \sum_{y \in S_{K}} p_{J K}(x, y) \log _{b} \frac{1}{p_{J K}(x, y)} .
$$

THEOREM 3.1. With the above assumptions, we have:

(i) $\log _{b}|\mathscr{I} \times \mathscr{L}|-H_{b}(X, Y) \geq P_{J K}\left[\log _{b}|J \times K|-H_{b}\left(X_{J}, Y_{K}\right)\right] \geq 0$;

(ii) if $(J, M)$ and $(K, N)$ provide proper decompositions of $\mathscr{I}$ and $\mathscr{L}$ respectively, then

$$
\log _{b}|\mathscr{I} \times \mathscr{L}|-H_{b}(X, Y) \geq \sum_{A=J, M} \sum_{B=K, N} P_{A B}\left[\log _{b}|A \times B|-H_{b}\left(X_{A}, Y_{B}\right)\right] \geq 0 .
$$

Proof. (i) Setting $f(\cdot)=-\log _{b}(\cdot), p_{i}=p(x, y)$ and $\xi_{i}=1 / p(x, y)$ in Theorem 1.1 , we obtain

$$
\begin{aligned}
\sum_{x} \sum_{y} p(x, y) \log _{b} p(x, y)+\log _{b}|J \times K| \\
\quad=\log _{b}|\mathscr{I} \times \mathscr{L}|-H_{b}(X, Y) \\
\quad \geq \sum_{x \in R_{J}} \sum_{y \in S_{K}} p(x, y) \log _{b} p(x, y)+\log _{b}|J \times K| \\
\quad=P_{J K}\left[\log _{b}|J \times K|+\sum_{x \in R_{J}} \sum_{y \in S_{K}} p_{J K}(x, y) \log _{b} p_{J K}(x, y)\right] \\
\quad=P_{J K}\left[\log _{b}|J \times K|-H_{b}\left(X_{J}, Y_{K}\right)\right] .
\end{aligned}
$$

(ii) The set $\mathscr{I} \times \mathscr{L}$ is the union of four disjoint sets:

$$
\mathscr{I} \times \mathscr{L}=(J \times K) \cup(M \times N) \cup(J \times N) \cup(M \times K) .
$$


On putting $f(\cdot)=-\log _{b}(\cdot), p_{i}=p(x, y)$ and $\xi_{i}=1 / p_{i}$ in Theorem 1.1, we get

We have

$$
F\left(-\log _{b}(\cdot), \mathscr{I} \times \mathscr{L}\right) \geq \sum_{A=J, M} \sum_{B=K, N} F\left(-\log _{b}(\cdot), A \times B\right) .
$$

$$
\begin{aligned}
F(- & \left.\log _{b}(\cdot), \mathscr{I} \times \mathscr{L}\right) \\
& =P_{\mathscr{I} \mathscr{L}} \log _{b}\left(\frac{1}{P_{\mathscr{I} \mathscr{L}}}|R \times S|\right)-\sum_{x \in R} \sum_{y \in S} p(x, y) \log _{b} \frac{1}{p(x, y)} \\
& =\log _{b}|\mathscr{I} \times \mathscr{L}|-H_{b}(X, Y) .
\end{aligned}
$$

Also for $A=J, M$ and $B=K, N$ we have

$$
\begin{aligned}
F(- & \left.\log _{b}(\cdot), A \times B\right) \\
& =P_{A B} \log _{b}\left(\frac{1}{P_{A B}}\left|R_{A} \times S_{B}\right|\right)-\sum_{x \in R_{A}} \sum_{y \in S_{B}} p(x, y) \log _{b} \frac{1}{p(x, y)} \\
& =\sum_{x \in R_{A}} \sum_{y \in S_{B}} p(x, y) \log _{b} p(x, y)+\log _{b}\left|R_{J} \times S_{K}\right| \\
& =P_{A B}\left[\log _{b}|A \times N|+\sum_{x \in R_{A}} \sum_{y \in S_{B}} p_{A B}(x, y) \log _{b} p_{A B}(x, y)\right] \\
& =P_{A B}\left[\log _{b}|A \times B|-H_{b}\left(X_{A}, Y_{B}\right)\right]
\end{aligned}
$$

since $p(x, y)=P_{A B} \cdot p_{A B}(x, y)$ and $\sum_{x \in R_{A}} \sum_{y \in S_{B}} p_{A B}(x, y)=1$. Equations (3.3)(3.5) taken together imply part (ii) of the theorem.

Theorem 3.1 (ii) leads directly to the following corollary.

COROLLARY 3.2. With the above assumptions we have

$$
\begin{aligned}
& \log _{b}|\mathscr{I} \times \mathscr{L}|-H_{b}(X, Y) \\
& \quad=\max \left\{P_{J K}\left[\log _{b}|J \times K|-H_{b}\left(X_{J}, Y_{K}\right)\right] \mid J \times K \subseteq \mathscr{I} \times \mathscr{L}, J, K \neq \emptyset\right\} \\
& \quad \geq \max _{\substack{i, j \in \mathscr{J} \\
m, n \in \mathscr{L}}}\left\{\left(p_{i m}+p_{i n}+p_{j m}+p_{j n}\right)\left[\log _{b} 4-H_{b}\left(X_{[i, j]}, Y_{[m, n]}\right)\right]\right\} \geq 0 .
\end{aligned}
$$

A result similar to Corollary 2.6 can be established for joint entropy.

COROLlaRY 3.3. Suppose $(J, M),(K, N)$ provide proper decompositions for $\mathscr{I}$ and $\mathscr{L}$ respectively. Then

$$
\max \left\{0, \log _{b} 4-\frac{1}{\ln b}\left[4 \sum_{A=J, M} \sum_{B=K, N} P_{A B}^{2}-1\right]\right\}
$$




$$
\begin{aligned}
& \leq H_{b}(X, Y)-\sum_{A=J, K} \sum_{B=K, N} P_{A B} H_{b}\left(X_{A}, Y_{B}\right) \\
& \leq \min \left\{\log _{b} 4, \log _{b}\left(\frac{|\mathscr{I} \times \mathscr{L}|}{\prod_{A=J, M} \prod_{B=K, N}|A \times B|^{P_{A B}}}\right)\right\} .
\end{aligned}
$$

Proof. First define the new random variable $Z$ by

$$
Z= \begin{cases}1 & \text { if }(X, Y) \in J \times K \\ 2 & \text { if }(X, Y) \in M \times N \\ 3 & \text { if }(X, Y) \in J \times N \\ 4 & \text { if }(X, Y) \in M \times K\end{cases}
$$

and put $q_{i}:=\operatorname{Pr}(Z=i)$ for $i=1,2,3,4$. Then $\sum_{i=1}^{4} \dot{q}_{i}=1$ and the entropy of $Z$ is

$$
H_{b}(Z)=\sum_{A=J, M} \sum_{B=K, N} P_{A B} \log _{b} \frac{1}{P_{A B}}
$$

which satisfies the usual entropy bound

$$
0 \leq H_{b}(Z) \leq \log _{b} 4 .
$$

Now

$$
\begin{aligned}
0 & \leq H_{b}(X, Y)-\sum_{A=J, M} \sum_{B=K, N} P_{A b} H_{b}\left(X_{A}, Y_{B}\right) \\
& =\sum_{(x, y) \in R \times S} p(x, y) \log _{b} \frac{1}{p(x, y)}-\sum_{A=J, M} \sum_{B=K, N} P_{A B} \sum_{(x, y) \in R_{A} \times S_{B}} p_{A B}(x, y) \log _{b} \frac{1}{p_{A B}(x, y)} \\
& =\sum_{(x, y) \in R \times S} p(x, y) \log _{b} \frac{1}{p(x, y)} \sum_{B=K, N} P_{A B} \sum_{(x, y) \in R_{A} \times S_{B}} p_{A B}(x, y) \log _{b} \frac{P_{A B}}{p(x, y)} \\
& =\sum_{A=J, M} \sum_{B=K, N} \log _{b} \frac{1}{P_{A B}} \sum_{(x, y) \in R_{A} \times S_{B}} p(x, y)=H_{b}(Z) .
\end{aligned}
$$

Next, we rewrite (3.1) as

$$
\begin{aligned}
H_{b}(Z) & =H_{b}(X, Y)-\sum_{A=J, M} \sum_{B=K, N} P_{A B} H_{b}\left(X_{A}, Y_{B}\right) \\
& \leq \log _{b}\left(\frac{|\mathscr{I} \times \mathscr{L}|}{\prod_{A=J, M} \prod_{B=K, N}|A \times B|^{P_{A B}}}\right) .
\end{aligned}
$$

The second inequality of the corollary thus follows from (3.6)-(3.8). Furthermore, applying Theorem 2.1 to $H_{b}(Z)$, we have

$$
0 \leq \log _{b} 4-H_{b}(Z) \leq \frac{1}{\ln b}\left[4 \sum_{A=J, M} \sum_{B=K, N} P_{A B}^{2}-1\right]
$$


which together with the fact that $H_{b}(Z) \geq 0$ yields the first inequality of the corollary.

In a similar way we may prove the following results for partial superadditivity and partial monotonicity.

COROLLARY 3.4. (i) Let $J \in 2^{\Im}, J \neq \emptyset$. Then

$$
\log _{b}|\mathscr{I} \times \mathscr{L}|-H_{b}(X, Y) \geq P_{J}\left[\log _{b}|J \times \mathscr{L}|-H_{b}\left(X_{J}, Y\right)\right] \geq 0 ;
$$

(ii) $\log _{b}|\mathscr{I} \times \mathscr{L}|-H_{b}(X, Y)=\max \left\{P_{J}\left[\log _{b}|J \times \mathscr{L}|-H_{b}\left(X_{J}, Y\right)\right] \mid J \in 2^{\mathscr{\sigma}}\right.$, $J \neq \emptyset\}$;

(iii) if $(J, M)$ constitutes a proper decomposition of $\mathscr{I}$, then

$$
\log _{b}|\mathscr{I} \times \mathscr{L}|-H_{b}(X, Y) \geq \sum_{A=J, M} P_{A}\left[\log _{b}|A \times \mathscr{L}|-H_{b}\left(X_{A}, Y\right)\right] \geq 0
$$

(iv) and

$$
0 \leq H_{b}(X, Y)-\sum_{A=J, M} P_{A} H_{b}\left(X_{A}, Y\right) \leq \min \left\{\log _{b} 2, \log _{b}\left[\frac{|\mathscr{I}|}{|J|^{P_{J}}|M|^{P_{M}}}\right]\right\} .
$$

Similar results can be obtained in the second variable.

\section{Some quasi-superadditivity / monotonicity properties of conditional entropy}

Let $X$ and $Y$ be two random variables having the ranges $R:=\left\{x_{i} \mid i \in \mathscr{I}\right\}$ and $S:=\left\{y_{i} \mid i \in \mathscr{L}\right\}$. For index subsets $J \in 2^{\mathcal{J}}, K \in 2^{\mathscr{L}}$, we define the conditional probability

$$
p_{J K}(x \mid y):=\frac{p_{J K}(x, y)}{p_{J K}(y)}
$$

where

$$
p_{J K}(y):=\sum_{x \in R_{J}} p(x, y) \quad \text { and } \quad \sum_{x \in R_{J}} p_{J K}(x \mid y)=1 \quad \forall y \in S_{K} .
$$

For $J \in \mathscr{I}$, the conditional entropy of $X_{J}$ given $Y$ is defined by

$$
H_{b}\left(X_{J} \mid Y\right):=\sum_{x \in R_{j}} \sum_{y \in S_{K}} p_{J \mathscr{L}}(x, y) \log _{b} \frac{1}{p_{J \mathscr{L}}(x \mid y)} .
$$

This is related to the joint entropy by

$$
H_{b}\left(X_{J} \mid Y\right)=H_{b}\left(X_{J}, Y\right)-H_{b}(Y) \text {. }
$$

For this partial conditional entropy, we have the following superadditivity and monotonicity properties. 
THEOREM 4.1. As an index set mapping, $P_{J}\left[\log _{b}|J|-H_{b}\left(X_{J} \mid Y\right)\right]$ is superadditive over $J$, that is, if $(J, M)$ provides a proper decomposition of $\mathscr{I}$, then

$$
\log _{b}|\mathscr{I}|-H_{b}(X \mid Y) \geq \sum_{A=J, M} P_{A}\left[\log _{b}|A|-H_{b}\left(X_{A} \mid Y\right)\right] \geq 0 .
$$

ProOF. From (4.1) with $X$ in place of $X_{J}$, Corollary 3.4 (iii) implies that

$$
\begin{aligned}
\log _{b}|\mathscr{I}|+\log _{b}|\mathscr{L}|-H_{b}(X \mid Y)-H_{b}(Y) \\
\quad \geq \sum_{A=J, M} P_{A}\left[\log _{b}|A|+\log _{b}|\mathscr{L}|-H_{b}\left(X_{A} \mid Y\right)-H_{b}(Y)\right],
\end{aligned}
$$

which is equivalent to

$$
\begin{aligned}
\log _{b}|\mathscr{I}|-H_{b}(X \mid Y) \geq & \sum_{A=J, M} P_{A}\left[\log _{b}|A|-H_{b}\left(X_{A} \mid Y\right)\right]+H_{b}(Y) \\
& -\log _{b}|\mathscr{L}|+\sum_{A=J, M} P_{A}\left[\log _{b}|\mathscr{L}|-H_{b}(Y)\right] \\
= & \sum_{A=J, M} P_{A}\left[\log _{b}|A|-H_{b}\left(X_{A} \mid Y\right)\right] .
\end{aligned}
$$

The following corollary follows directly from Theorem 4.1.

COROLLARY 4.2 .

$$
\begin{aligned}
\log _{b}|\mathscr{I}|-H_{b}(X \mid Y) & =\max _{\substack{J \subseteq \mathscr{S} \\
J \neq \emptyset}}\left\{P_{J}\left[\log _{b}|J|-H_{b}\left(X_{J} \mid Y\right)\right]\right\} \\
& \geq \max _{i, j \in \mathscr{J}}\left\{\left(p_{i}+p_{j}\right)\left[\log _{b} 2-H_{b}\left(X_{\{i, j\}} \mid Y\right)\right]\right\} \geq 0 .
\end{aligned}
$$

Similarly, the following result follows directly from Corollary 3.4 (iv).

COROLlaRY 4.3. Suppose $(J, M)$ is a proper decomposition of $\mathscr{I}$. Then

$$
0 \leq H_{b}(X \mid Y)-\sum_{A=J, M} P_{A} H_{b}\left(X_{A} \mid Y\right) \leq \min \left\{\log _{b} 2, \log _{b}\left[\frac{|\mathscr{I}|}{|J|^{P_{J}}|M|^{P_{M}}}\right]\right\} .
$$

Superadditivity and monotonicity properties similar to those of Theorem 3.1 and its corollaries do not in general hold for conditional entropy. However, for the partial conditional entropy

$$
H_{b}\left(X \mid Y_{K}\right):=\sum_{x \in R} \sum_{y \in S_{K}} p_{\mathscr{I}_{K}}(x, y) \log _{b} \frac{1}{p_{\mathscr{J} K}(x \mid y)}=H_{b}\left(X, Y_{K}\right)-H_{b}\left(Y_{K}\right),
$$

some quasi-superadditivity and quasi-monotonicity properties can be established for the second argument in $H_{b}(\cdot \mid \cdot)$. 
THEOREM 4.4. As a mapping on the index set $K, P_{K}\left[\log |\mathscr{I}|-H_{b}\left(X \mid Y_{K}\right)\right]$ is quasi-superadditive, that is, if $(K, N)$ is a proper decomposition of $\mathscr{L}$, then

$$
\log _{b}|\mathscr{I}|-H_{b}(X \mid Y) \geq \sum_{B=K, N} P_{B}\left[\log _{b}|\mathscr{I}|-H_{b}\left(X \mid Y_{B}\right)\right]+\log _{b}\left(\frac{|K|^{P_{K}}|N|^{P_{N}}}{|\mathscr{L}|}\right)
$$

PROOF. Using the (partial) superadditivity of the joint entropy function $H_{b}(\cdot, \cdot)$ in the second argument (cf. Corollary 3.4 (iii)), we have

$$
\log _{b}|\mathscr{I} \times \mathscr{L}|-H_{b}(X, Y) \geq \sum_{B=K, N} P_{B}\left[\log _{b}|\mathscr{I} \times B|-H_{b}\left(X, Y_{B}\right)\right] \geq 0,
$$

which is equivalent to

or

$$
\begin{aligned}
\log _{b}|\mathscr{I}|+\log _{b}|\mathscr{L}|-H_{b}(X \mid Y)-H_{b}(Y) \\
\quad \geq \sum_{B=K, N} P_{B}\left[\log _{b}|\mathscr{I}|+\log _{b}|B|-H_{b}\left(X \mid Y_{B}\right)-H_{b}\left(Y_{B}\right)\right]
\end{aligned}
$$

$$
\begin{aligned}
\log _{b}|\mathscr{I}|-H_{b}(X \mid Y) \geq & \sum_{B=K, N} P_{B}\left[\log _{b}|\mathscr{I}|-H_{b}\left(X \mid Y_{B}\right)\right] \\
& +H_{b}(Y)-\log _{b}|\mathscr{L}|+\sum_{B=K, N} P_{B}\left[\log _{b}|B|-H_{b}\left(Y_{B}\right)\right] .
\end{aligned}
$$

As $H_{b}(Y) \geq \sum_{B=K, N} P_{B} H_{b}\left(Y_{B}\right) \geq 0$ and $P_{K}+P_{N}=1$, the desired result follows.

THEOREM 4.5. Let $K \in 2^{\mathscr{L}}, K \neq \emptyset$. Then $P_{K}\left[\log |\mathscr{I}|-H_{b}\left(X \mid Y_{K}\right)\right]$ is quasimonotone as a mapping on the index set $K$, that is,

$$
\log _{b}|\mathscr{I}|-H_{b}(X \mid Y) \geq P_{K}\left[\log _{b}|\mathscr{I}|-H_{b}\left(X \mid Y_{K}\right)\right]+\log _{b}\left(\frac{|K|^{P_{K}}}{|\mathscr{L}|}\right)
$$

PROOF. Using the partial monotonicity of the joint entropy function $H_{b}(\cdot, \cdot)$ in the second argument (cf. Corollary 3.4 (i)), we have

$$
\begin{aligned}
\log _{b}|\mathscr{I}|+\log _{b}|\mathscr{L}|-H_{b}(X \mid Y)-H_{b}(Y) \\
\quad \geq P_{K}\left[\log _{b}|\mathscr{I}|+\log _{b}|K|-H_{b}\left(X \mid Y_{K}\right)-H_{b}\left(Y_{K}\right)\right],
\end{aligned}
$$

which is equivalent to

$$
\begin{aligned}
\log _{b}|\mathscr{I}|-H_{b}(X \mid Y) \geq & P_{K}\left[\log _{b}|\mathscr{I}|-H_{b}\left(X \mid Y_{K}\right)\right] \\
& +H_{b}(Y)-\log _{b}|\mathscr{L}|+P_{K} \log _{b}|K|-P_{K} H_{b}\left(Y_{K}\right) .
\end{aligned}
$$

Since $H_{b}(Y) \geq P_{K} H_{b}\left(Y_{K}\right)+P_{\mathscr{L} \backslash K} H_{b}\left(Y_{\mathscr{L} \backslash K}\right) \geq P_{K} H_{b}\left(Y_{K}\right)$, the conclusion of the corollary follows. 
Given $J \subseteq \mathscr{I}, K, J \subseteq \mathscr{L}, J, K \neq \emptyset$, we define the conditional entropy of $X_{J}$ given $Y_{K}$ by

$$
H_{b}\left(X_{J} \mid Y_{K}\right):=\sum_{x \in R_{J}} \sum_{y \in S_{K}} p_{J K}(x, y) \log _{b} \frac{1}{p_{J K}(x \mid y)}=H_{b}\left(X_{J}, Y_{K}\right)-H_{b}\left(Y_{K}\right)
$$

THEOREM 4.6. Let $(J, M),(K, N)$ be respectively proper decompositions of $\mathscr{I}$ and $\mathscr{L}$. Then

$$
\begin{aligned}
\log _{b}|\mathscr{I}|-H_{b}(X \mid Y) \geq & \sum_{A=J, M} \sum_{B=K, N} P_{A B}\left[\log _{b}|A|-H_{b}\left(X_{A} \mid Y_{B}\right)\right] \\
& +\log _{b}\left(\frac{|K|^{P_{K}}|N|^{P_{N}}}{|\mathscr{L}|}\right) .
\end{aligned}
$$

PROOF. From the superadditivity property of $H_{b}(X, Y)(c f$. Theorem 3.1 (ii)) and (4.1) with $Y$ replaced by $Y_{K}$, we obtain

$$
\begin{aligned}
\log _{b}|\mathscr{I} \times \mathscr{L}|-H_{b}(X \mid Y)-H_{b}(Y) \\
\geq \sum_{A=J, M} \sum_{B=K, N} P_{A B}\left[\log _{b}|A \times B|-H_{b}\left(X_{A} \mid Y_{B}\right)-H_{b}\left(Y_{B}\right)\right],
\end{aligned}
$$

which is equivalent to

$$
\begin{aligned}
\log _{b}|\mathscr{I}|-H_{b}(X \mid Y) \geq & \sum_{A=J, M} \sum_{B=K, N} P_{A B}\left[\log _{b}|A|-H_{b}\left(X_{A} \mid Y_{B}\right)\right]+H_{b}(Y) \\
& -\log _{b}|\mathscr{L}|+\sum_{A=J, M} \sum_{B=K, N} P_{A B}\left[\log _{b}|B|-H_{b}\left(Y_{B}\right)\right]
\end{aligned}
$$

Since for $B=K, N$ we have

$$
\sum_{A=J, M} P_{A B} \log _{b}|B|=P_{B} \log _{b}|B|
$$

and

$$
\sum_{A=J, M} P_{A B} H_{b}\left(Y_{B}\right)=P_{B} H_{b}\left(Y_{B}\right)
$$

we deduce that

$$
H_{b}(Y)-\sum_{B=K, N} P_{B} H_{b}\left(Y_{B}\right)+\log _{b}\left(\frac{|K|^{P_{K}}|N|^{P_{N}}}{|\mathscr{L}|}\right) \geq \log _{b}\left(\frac{|K|^{P_{K}}|N|^{P_{N}}}{|\mathscr{L}|}\right),
$$

as $H_{b}(Y) \geq \sum_{B=K, N} P_{B} H_{b}\left(Y_{B}\right)$. The conclusion of the theorem follows from (4.2) and (4.3).

The following quasi-monotonicity property of conditional entropy follows from the monotonicity of joint entropy (see Theorem 3.1 (i)). The proof is similar to that of Theorem 4.5. 
COROLlary 4.7. Let $K \in 2^{\mathscr{L}}, K \neq \emptyset, J \in 2^{\mathscr{I}}, J \neq \emptyset$. Then we have

$$
\log _{b}|\mathscr{I}|-H_{b}(X \mid Y) \geq P_{J K}\left[\log _{b}|J|-H_{b}\left(X_{J} \mid Y_{K}\right)\right]+\log _{b}\left(|K|^{P_{K}} /|\mathscr{L}|\right)
$$

\section{Extension to mutual information}

The marginal probability distributions of two random variables $X, Y$ with joint probability distribution $p(x, y)$ are respectively

$$
p(x)=\sum_{y} p(x, y) \quad \text { and } \quad q(y)=\sum_{x} p(x, y),
$$

so that $\sum_{x} p(x)=1$ and $\sum_{y} q(y)=1$. Here $\sum_{x}, \sum_{y}$ are by default respectively summations over the full ranges of $R$ and $S$. Define the mutual information of $X$ and $Y$ by

$$
I_{b}(X, Y):=\sum_{x \in R} \sum_{y \in S} p(x, y) \log _{b} \frac{p(x, y)}{p(x) q(y)}
$$

For some index subsets $J \in 2^{\mathscr{J}}$ and $K \in 2^{\mathscr{L}}$, we define the pair of new random variables $X_{J}$ and $Y_{K}$ having ranges in $R_{J}:=\left\{x_{i}, i \in J\right\}$ and $S_{K}:=\left\{y_{i}, i \in K\right\}$ respectively.

The mutual information of $X_{J}$ and $Y_{K}$ is defined as

$$
I_{b}\left(X_{J}, Y_{K}\right):=\sum_{x \in J} \sum_{y \in K} p_{J K}(x, y) \log _{b} \frac{p_{J K}(x, y)}{p_{J}(x) q_{K}(y)}
$$

THEOREM 5.1. With the above assumptions,

(i) $I_{b}(X, Y) \geq P_{J K} I_{b}\left(X_{J}, Y_{K}\right)$;

(ii) if $(J, M),(K, N)$ are proper decompositions of $\mathscr{I}$ and $\mathscr{L}$ respectively, then

$$
I_{b}(X, Y) \geq \sum_{A=J, M} \sum_{B=K, N} P_{A B} I_{b}\left(X_{A}, Y_{B}\right)
$$

PROOF. (i) Set $f(\cdot)=-\log _{b}(\cdot), p_{i}=p(x, y)$ and $\xi_{i}=p(x) q(y) / p(x, y)$ in Theorem 1.1 to obtain

$$
\begin{aligned}
& \log _{b}\left(\sum_{x} \sum_{y} p(x, y) \frac{p(x) q(y)}{p(x, y)}\right)-\sum_{x} \sum_{y} p(x, y) \log _{b}\left(\frac{p(x) q(y)}{p(x, y)}\right) \\
& \quad=I_{b}(X, Y)
\end{aligned}
$$




$$
\begin{aligned}
\geq & P_{J K} \log _{b}\left(\frac{1}{P_{J K}} \sum_{x \in R_{J}} \sum_{y \in S_{K}} p(x, y) \frac{p(x) q(y)}{p(x, y)}\right) \\
& -\sum_{x \in R_{J}} \sum_{y \in S_{K}} \frac{p(x, y)}{P_{J K}} \log _{b}\left(\frac{p(x) q(y)}{p(x, y)}\right) \\
= & P_{J K}\left(\log _{b}\left[\sum_{x \in R} \sum_{y \in S_{K}} p(x) q(y)\right]-\sum_{x \in R_{J}} \sum_{y \in S_{K}} p_{J K}(x, y) \log _{b}\left(\frac{p(x) q(y)}{p_{J K}(x, y)}\right)\right) \\
= & P_{J K}\left[\sum_{x \in R_{J}} \sum_{y \in S_{K}} p_{J K}(x, y) \log _{b} \frac{p_{J K}(x, y)}{p_{J}(x) q_{K}(y)}\right]=P_{J K} I_{b}\left(X_{J}, Y_{K}\right) .
\end{aligned}
$$

(ii) From the decomposition (3.2), an application of Theorem 1.1 leads to

$$
\begin{aligned}
\mathscr{F}( & \left.-\log _{b}(\cdot), \mathscr{I} \times \mathscr{L}, p(x, y), \frac{p(x) q(y)}{p(x, y)}\right) \\
& \geq \sum_{A=I, M} \sum_{B=K, N} \mathscr{F}\left(-\log _{b}(\cdot), A \times B, p(x, y), \frac{p(x) q(y)}{p(x, y)}\right)
\end{aligned}
$$

Furthermore, from the proof in (i) above, we have

$$
\begin{aligned}
& \mathscr{F}\left(-\log _{b}(\cdot), \mathscr{I} \times \mathscr{L}, p(x, y), \frac{p(x) q(y)}{p(x, y)}\right)=I_{b}(X, Y), \\
& \mathscr{F}\left(-\log _{b}(\cdot), A \times B, p(x, y), \frac{p(x) q(y)}{p(x, y)}\right)=P_{A B} I_{b}\left(X_{A}, Y_{B}\right)
\end{aligned}
$$

for $A=M, J$ and $B=K, N$. The desired result follows from (5.1) and (5.2).

The following corollary follows immediately from Theorem 5.1 (i).

COROLLARY 5.2. With the above assumptions, we have

$$
\begin{aligned}
I_{b}(X, Y) & =\max _{\substack{\emptyset \neq J \subset \Phi \\
\emptyset \neq K \subset \mathscr{L}}}\left\{P_{J K} I_{b}(X, Y)\right\} \\
& \geq \max _{\substack{i, j \in \mathscr{S} \\
m, n \in \mathscr{S}}}\left\{\left(P_{i, m}+P_{j, m}+P_{i, n}+P_{j, n}\right) I_{b}\left(X_{\{i, j\}}, Y_{\{m, n\}}\right)\right\} \geq 0 .
\end{aligned}
$$

A sharper bound on the various mutual information measures can be established as follows.

THEOREM 5.3. Suppose $(J, M),(K, N)$ are respectively proper decompositions of $\mathscr{I}$ and $\mathscr{L}$. Then

$$
\max \left\{0, \sum_{(A, B)=(J, M),(K, N)} \max \left\{0, \log _{b} 2-\frac{1}{\ln b}\left(P_{A}-P_{B}\right)^{2}\right\}\right.
$$




$$
\begin{aligned}
& -\min \left\{\log _{b} 4, \log _{b}\left(\frac{|\mathscr{I} \times \mathscr{L}|}{\prod_{A=J, M} \prod_{B=K, N}|A \times B|^{P_{A B}}}\right)\right\} \\
\leq & I_{b}(X, Y)-\sum_{A=J, M} \sum_{B=K, N} P_{A B} I_{b}\left(X_{A}, Y_{B}\right) \\
\leq & \sum_{(A, B)=(J, M),(K, N)} \min \left\{\log _{b} 2, \log _{b}\left[\frac{|\mathscr{I}|}{\left.\left.|A|^{P_{A}|B|^{P_{B}}}\right]\right\}}\right.\right. \\
& -\max \left\{0, \log _{b} 4-\frac{1}{\ln b}\left[4 \sum_{A=J, M} \sum_{B=K, N} P_{A B}^{2}-1\right]\right\} .
\end{aligned}
$$

PROOF. From the identity $I_{b}(X, Y)=H_{b}(X)+H_{b}(Y)-H_{b}(X, Y)$ we derive

$$
\begin{aligned}
C:=I_{b}(X, Y)-\sum_{A=J, M} \sum_{B=K, N} P_{A B} I_{b}\left(X_{A}, Y_{B}\right) \\
=H_{b}(X)+H_{b}(Y)-H_{b}(X, Y)-\sum_{A=J, M} \sum_{B=K, N} P_{A B}\left(H_{b}\left(X_{A}\right)+H_{b}\left(Y_{B}\right)-H_{b}\left(X_{A}, Y_{B}\right)\right) \\
=H_{b}(X)-\sum_{A=J, M} P_{A} H_{b}\left(X_{A}\right)+H_{b}(Y)-\sum_{B=K, N} P_{B} H_{b}\left(Y_{B}\right) \\
\quad-H_{b}(X, Y)+\sum_{B=K, N} P_{J B} H_{b}\left(X_{J}, Y_{B}\right)-\sum_{B=K, N} P_{M B} H_{b}\left(X_{M}, Y_{B}\right) .
\end{aligned}
$$

From Corollary 2.6, we have

$$
\begin{aligned}
\alpha_{1} & :=\max \left\{0, \log _{b} 2-\frac{1}{\ln b}\left(P_{J}-P_{M}\right)^{2}\right\} \leq H_{b}(X)-\sum_{A=J, M} P_{A} H_{b}\left(X_{A}\right) \\
& \leq \min \left\{\log _{b} 2, \log _{b}\left[\frac{|\mathscr{I}|}{|J|^{P_{J}}|M|^{P_{M}}}\right]\right\}:=\beta_{1},
\end{aligned}
$$

and

$$
\begin{aligned}
\alpha_{2} & :=\max \left\{0, \log _{b} 2-\frac{1}{\ln b}\left(P_{K}-P_{N}\right)^{2}\right\} \leq H_{b}(Y)-\sum_{B=K, N} P_{B} H_{b}\left(Y_{B}\right) \\
& \leq \min \left\{\log _{b} 2, \log _{b}\left[\frac{|\mathscr{L}|}{|K|^{P_{K}}|N|^{P_{N}}}\right]\right\}:=\beta_{2} .
\end{aligned}
$$

From Corollary 3.3, we have

$$
\begin{aligned}
\alpha_{3} & :=\max \left\{0, \log _{b} 4-\frac{1}{\ln b}\left[4 \sum_{A=J, M} \sum_{B=K, N} P_{A B}^{2}-1\right]\right\} \\
& \leq H_{b}(X, Y)-\sum_{A=J, M} \sum_{B=K, N} P_{A B} H_{b}\left(X_{A}, Y_{B}\right)
\end{aligned}
$$




$$
\leq \min \left\{\log _{b} 4, \log _{b}\left(\frac{|\mathscr{I} \times \mathscr{L}|}{\prod_{A=J, M} \prod_{B=K, N}|A \times B|^{P_{A B}}}\right)\right\}:=\beta_{3} .
$$

Putting these results together, we have

$$
\alpha_{1}+\alpha_{2}-\beta_{3} \leq C \leq \beta_{1}+\beta_{2}-\alpha_{3}
$$

and the theorem is proved.

\section{Acknowledgements}

The first author acknowledges the financial support from The University of Western Australia during his visit when this work was completed.

\section{References}

[1] S. S. Dragomir and C. J. Goh, "A counterpart of Jensen's discrete inequality for differentiable convex mappings and applications in information theory", Math. Comp. Modeling 24 (1996) 1-11.

[2] S. S. Dragomir, J. Pečarić and L. E. Persson, "Properties of some functionals related to Jensen's inequality", Acta Math. Hung. 70 (1996) 129-143.

[3] R. J. McEliece, The theory of information and coding (Addison Wesley, Reading, 1977). 